\title{
Aportaciones a la flora de la comunidad de Madrid (España)
}

\author{
José Araújo Díaz de Terán ${ }^{1}$, Ginés López González², Álvaro Izuzquiza Ibáñez de Aldecoa ${ }^{3}$, Daniel \\ Sánchez Mata ${ }^{4}$ \& Juan Manuel Martínez Labarga ${ }^{*}$ \\ 1. Casa Forestal "las Gariñas", ctra de Buitrago a Mangirón, pk 1,5, Buitrago de Lozoya, Madrid - 28730 \\ ${ }^{2} \mathrm{C} /$ Junco 23, El Encinar del Alberche, Villa del Prado, Madrid - 28630 \\ ${ }^{3} \mathrm{C} /$ Costa Rica 16, Madrid - 28016 \\ ${ }^{4}$ Departamento Farmacología, Farmacognosia y Botánica (Unidad de Botánica), Facultad de Farmacia. Universidad \\ Complutense de Madrid, Madrid - 28040 \\ ${ }^{5}$ Unidad docente de Botánica, Escuela Técnica Superior de Ingeniería de Montes, Forestal y del Medio Natural. \\ Departamento de Sistemas y Recursos Naturales. Universidad Politécnica de Madrid, Madrid - 28040
}

\section{Correspondencia}

J.M. Martínez Labarga

e-mail: juanmanuel.martinez@upm.es

Recibido: 19 noviembre 2019

Aceptado: 18 julio 2020

Publicado on-line: 29 julio 2020

Editado por: Gabriel Blanca López

\section{Resumen}

Se aportan 7 nuevas citas de plantas para el territorio de Madrid dando continuidad a trabajos previos de flora realizados por los mismos autores en dicha región. De cada taxon se aporta información corológica general y de Madrid, testimonios de herbario, así como información sobre su conservación en algunas de las citas.

Palabras clave: Flora, corología, conservación, península ibérica.

\section{Abstract}

Contributions to the flora of the Community of Madrid (Spain)

7 new citations of plants are available for the territory of Madrid as a continuation of previous works on plants of the region performed by the same authors. General and chorological information is provided for each taxon, as well as herbarium testimonies, and information on their conservation in some of the citations.

Key words: Flora, chorology, conservation, iberian peninsula.
En este trabajo se proporciona información de 7 nuevas citas de plantas que no estaban debidamente documentadas para el territorio de Madrid, indicando los testimonios de herbario. Destacan Eryngium corniculatum Lam. y Romulea ramiflora Ten. que constituyen novedad provincial, y se confirman Epipactis helleborine (L.) Crantz y Sanguisorba hybrida (L.) Font Quer. Para el resto de especies se confirman localidades que no se encontraban disponibles en los medios más habituales para la consulta corológica. En algunos casos se muestra el grado de amenaza de estas plantas en el territorio según los criterios de la UICN.

En la línea de trabajo en la que estamos inmersos desde hace años sobre el conocimiento de la flora madrileña, y dentro de los estudios realizados en la región de la cuenca del Tajo (Martínez Labarga, 2014), se aportan novedades a la flora de la Comunidad de Madrid como continuación de trabajos previos publicados (Martínez Labarga, 2016, 2018; Martínez Labarga et al., 2017), en este caso con especies que se localizan prioritariamente en el sector suroccidental provincial. Se han recopilado y puesto en común informaciones aportadas por diferentes investigadores y profesionales del medio natural de varios organismos e instituciones.

Para contrastar la información taxonómica y corológica, se han consultado los datos de los herbarios $A B H$, JACA, MA, MACB, MAF y VAL (acrónimos según Thiers \& Tulig, 2019), los datos disponibles online (Anthos, 2019; Biodiversidadvirtual.org, 2019; Gbif, 2019; Real Jardín Botánico-CSIC: Colecciones, 2018; Sivim, 2018; The Plant List, 2013) y los catálogos disponibles (Cutanda, 1861; Abajo et al., 1982; Morales Valverde, 2003; López Jiménez, 2007; Grijalbo, 2016 b). Las coordenadas UTM se muestran en el datum ETRS89. Además, se comenta si algunos taxones tratados pueden tener interés conservacionista.

\section{Clematis flammula $\mathrm{L}$.}

ESPAÑA, Madrid: Madrid, Moncloa, orillas del río Manzanares, junto a la tapia del Club de Campo, frente a la depuradora de Viveros, 30TVK3678, 590 $\mathrm{m}$., en soto arbóreo mixto y herbazal nitrófilo sobre sustrato arenoso aluvial, 26-XII-2016, J.M. Martínez Labarga (20192-3/2016-12-26JML) (MAF 178102); ibidem, 21-X-2018, J.M. Martínez Labarga (212141/2018-10-21JML) (MAF 177423).

Región mediterránea y Asia Occidental; en la península ibérica es claramente mediterránea, con algunas localidades en la cornisa cántabra. En Martínez Labarga (2014) se consideró poco probable la presencia de esta planta en Madrid, a pesar de que había sido confirmada para Flora iberica (Fernández Carvajal, 1986: 269) y posteriormente por López Jiménez (2007: 87). La revisión de herbarios no ha 
permitido detectar ejemplares madrileños. Por eso la presencia de una buena población en la ribera del Manzanares, constituye una novedad florística importante, máxime porque se ha localizado en un hábitat poco propicio para la especie, hecho que hace pensar en un asilvestramiento antiguo a partir del antiguo huerto de Migas Calientes, que fue la primera localización del Jardín Botánico de Madrid. La consulta de los archivos de las plantas empleadas en dicho jardín (Inventario, 1771: 12) confirman que se utilizó la Clematis foliis pinnatifidis flore albo, que sin duda correspondería con la Clematis flammula y a partir de aquí podría haberse producido el asilvestramiento que perdura en nuestros días.

\section{Epipactis helleborine (L.) Crantz subsp. helleborine}

ESPAÑA, Madrid: Cenicientos, umbría de la Peña de Cenicientos, 30TUK7459, $930 \mathrm{~m}$, en ambiente de castañar, 30-VI-2018, G. López González (Fig. 1).

Epipactis helleborine es una orquídea típica del interior de bosques caducifolios en climas templados. Se distribuye por Eurasia y en la península ibérica por el tercio norte (Crespo, 2005: 30). En Madrid es muy rara y estaba puesta en entredicho (Crespo, 2005) porque algunas citas previas tras la consulta del material de herbario se habían reasignado a Epipactis tremolsii Pau (véase MA 539821). Con esta aportación se confirma y corrobora la cita previa de
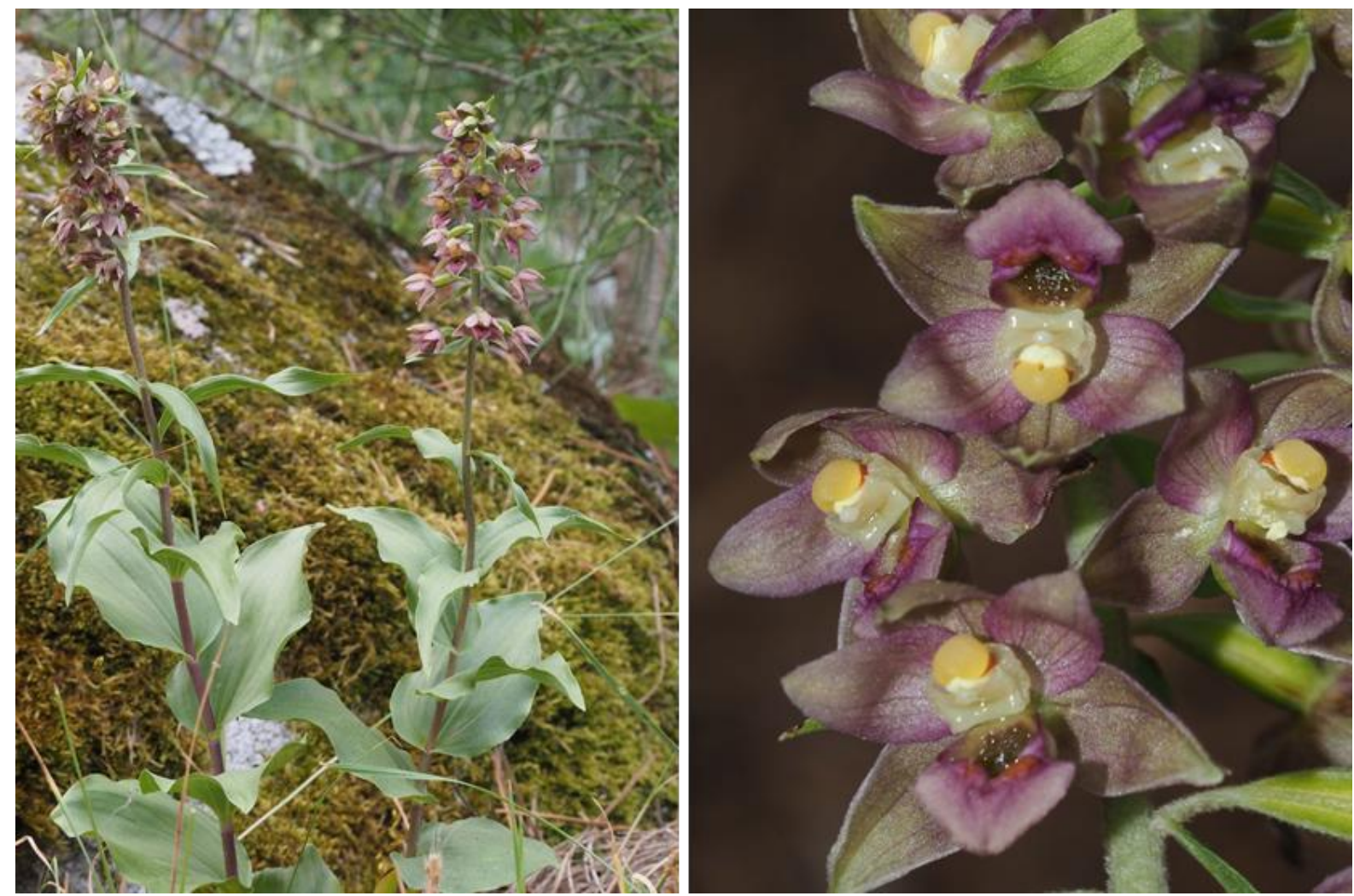

Figura 1. Epipactis helleborine en Cenicientos (Madrid). A- hábito, B - inflorescencia

Figure 1. Epipactis helleborine in Cenicientos (Madrid). A- habit, B-inflorescence.

Cebolla Lozano \& Rivas Ponce (1994: 163) en esta misma localidad. Estas citas se completarían en Madrid con la de Baonza Díaz (2007) para la Dehesa Vieja de Bustarviejo.

Respecto a la conservación de la especie, se debería incluir para Madrid entre su flora protegida, por ser una orquídea muy escasa de ambientes forestales bien conservados.

\section{Eryngium corniculatum Lam.}

ESPAÑA, Madrid: Hoyo de Manzanares, Lanchas de Castilla, al norte de la urbanización La Berzosa, en charca temporal, 30TVK2096, 975 m., 25-VII-2018, J. Araújo Díaz de Terán \& J.M. Martínez Labarga (21113-1/2018-07-25JML) (MAF 177426) (Fig. 2).

Eryngium corniculatum se distribuye en el oeste de la península ibérica, Cerdeña y Norte de
Marruecos (Gbif, 2019), habita lagunas y charcas temporales (Cirujano Bracamonte \& Medina Domingo, 2002: 133; Nieto Feliner, 2003: 60) y no se han detectado registros de la especie en Madrid. Se han localizado unos 250 ejemplares en el interior del lecho de una charca temporal ya desecada por el estío, con tendencia a ocupar la zona central más profunda. Junto a Eryngium corniculatum encontramos otras especies afines a charcas temporales como Eleocharis palustris (L.) Roem. \& Schult., Lythrum borysthenicum (Schrank) Litv., Juncus tenageia Ehrh. ex L. fil., J. pygmaeus Rich. ex Thuill., J. bufonius L., J. articulatus L., Cicendia filiformis (L.) Delarbre y Crassula vaillantii (Willd.) Roth entre otras, que proporcionan riqueza florística al enclave y que nos permiten encuadrar esta comunidad en el hábitat prioritario de "Lagunas y 
charcas temporales mediterráneas" (Bartolomé et al., 2005: 94), que se encuentra incluido en la Directiva 92/43/CEE relativa a la conservación de los hábitats naturales y de la fauna y flora silvestres (DOUE, 1992) en su anexo I bajo el código 3170*. En definitiva este enclave y esta especie deberían tener asegurada su conservación y se debería incluir en el catálogo de especies amenazadas madrileñas, porque según los criterios UICN (2012) estaría clasificada en peligro crítico.

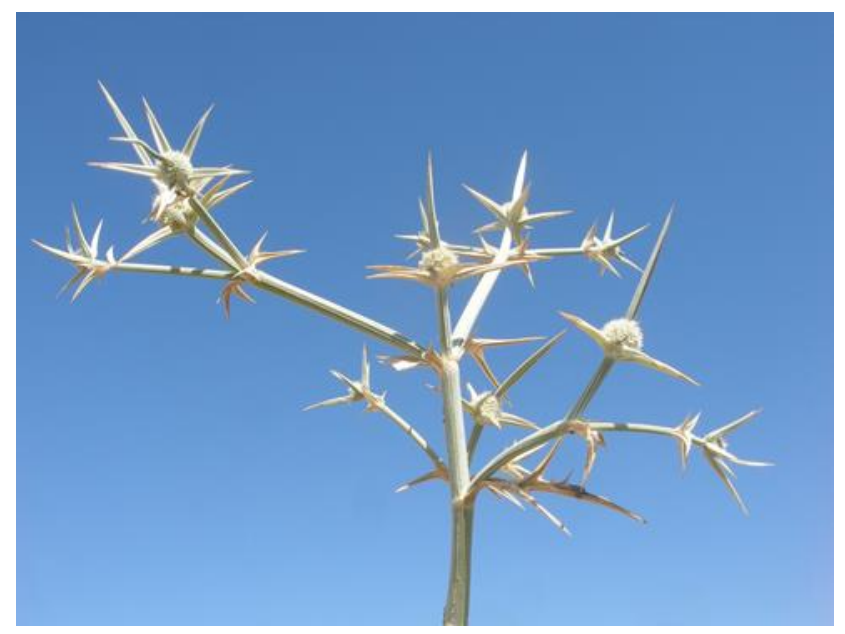

Figura 2. Eryngium corniculatum en Hoyo de Manzanares (Madrid).

Figure 2. Eryngium corniculatum in Hoyo de Manzanares (Madrid).
Glebionis segetum (L.) Fourr.

[= Chrysanthemum segetum L.]

ESPAÑA, Madrid: Vallecas, mayo de 1892, Mas Guindal (MAF 63302); Villa del Prado, cunetas, 15-VI1984, D. Sánchez Mata (MAF 118416) [sub Chrysanthemum segetum L.]; ibidem, márgenes de la carretera M-540 muy cerca del arroyo Arrofresno, 30TUK8956, 470 m., bordes de cultivos, 11-IV-2017, J. Araújo Díaz de Terán (Fig. 3); Madrid, Moncloa, Ciudad Universitaria, inmediaciones de la facultad de C.C. Geológicas, 30TVK3877, 640 m., en herbazal ruderal en zona ajardinada, 25-II-2020, J.M. Martínez Labarga (21588-1/2020-02-25JML) (MAF 178103).

Europa, Macaronesia, $\mathrm{N}$ de África y W de Asia, naturalizado en diversas zonas (Gbif, 2019); en la península ibérica es una planta de la mayoría de las provincias costeras mediterráneas y de las regiones occidentales menos frías, abundante hacia el SW, que desaparece hacia el interior y se enrarece en el norte. Para Madrid, López Jiménez (2007: 291), la consideró extinguida y solo recogió la información del pliego de Vallecas [MAF 63302, leg. Mas Guindal, 1892]. Posteriormente ha sido citada por Benedí (2019: 1979) y Grijalbo (2016 a: 318; 2016 b) que la sitúa en dos puntos de la localidad de Villa del Prado aquí aportada. No nos consta que haya otros testimonios de herbario, por lo que las localidades aquí presentadas corroboran la especie para Madrid, cerca del límite provincial con Toledo. Estas localidades conforman el límite de distribución oriental por el centro peninsular.
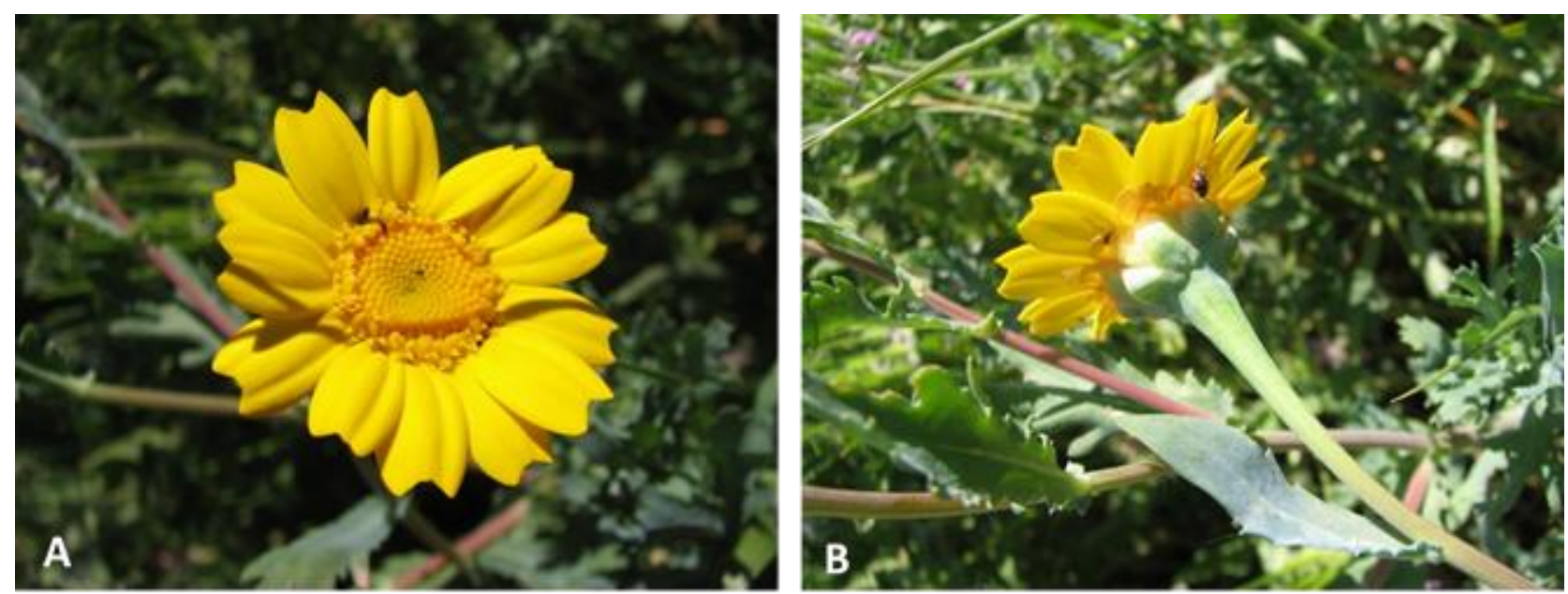

Figura 3. Glebionis segetum en Villa del Prado (Madrid). A- capítulo vista frontal, B - capítulo vista lateral.

Figure 3. Glebionis segetum in Villa del Prado (Madrid). A- front view capitule, B-side view capitule.

\section{Romulea ramiflora Ten. subsp. ramiflora}

ESPAÑA, Madrid: Villa del Prado, Dehesa del Alamar, al sur del hospital Virgen de la Poveda, 30TUK9155, 460 m., en pastizal en claro de encinar adehesado, 30-III-2015, J. Araújo Díaz de Terán \& J.M. Martínez Labarga (19250-2/2015-03-30JML) (MAF 178104); ibidem, 23-II-2016, J. Araújo Díaz de Terán (MAF 176142) (Fig. 4); Villa del Prado, al sur del Cerro Vacas, entre el Arroyo del Molino y el Arroyo Arrofresno, 30TUK9054, 440 m., or. SE, en claros de encinar arbustivo con retamar-pastizal, 15-III-2018, J.
Araújo Díaz de Terán, G. López González \& J.M. Martínez Labarga (20597-1/2018-03-15JML) (MAF 178105).

Geófito mediterráneo que se distribuye en la península ibérica por el cuadrante suroccidental y en las costas, exceptuando la cantábrica (Anthos, 2019; Gbif, 2019; Cardiel, 2013: 472). La localidad aquí aportada supone la primera cita provincial para la especie, encontrada en dos parajes del suroeste de la Comunidad de Madrid, a escasos metros de la provincia de Toledo en suelos bien drenados de 
antiguas terrazas fluviales del Alberche. Las dos localizaciones se hallan en la subprovincia corológica luso-extremadurense, hecho que se constata por la presencia de Drimia maritima (L.) Stearn, considerado elemento termófilo muy común en ese ámbito corológico.

Respecto a la conservación de la especie, consideramos que al menos debería incluirse en el catálogo madrileño de especies amenazadas, ya que según los criterios UICN (2012) entraría en la categoría de en peligro de extinción.

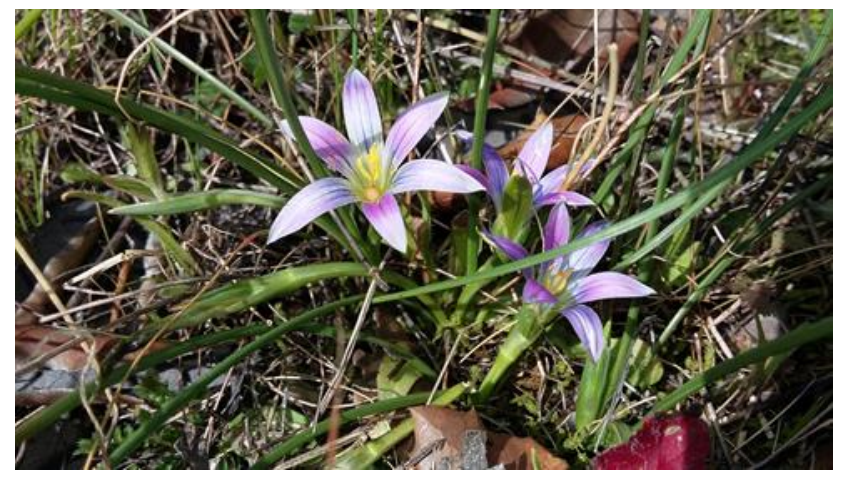

Figura 4. Romulea ramiflora en Villa del Prado (Madrid). Figure 4. Romulea ramiflora in Villa del Prado (Madrid).

Sanguisorba hybrida (L.) Font Quer

ESPAÑA, Madrid: Cadalso de los Vidrios, Arroyo Labros, en el límite de provincia, 30TUK8262, 705 m, en herbazal-matorral en la orilla del arroyo en ambiente de pinar de Pino piñonero sobre granitos, 21-VI-2018, G. López González (MAF 177429) (Fig. 5).

Endemismo del cuadrante suroccidental ibérico (Navarro \& Muñoz Garmendia, 1998: 379), típica de alcornocales y manchas sobre suelos de naturaleza silícea; no se había vuelto a citar en Madrid desde Cutanda (1861: 282) "Escorial", [sub Poterium hybridum L.]. Con la localidad aquí aportada se confirma para la provincia de Madrid y se corrobora la hipótesis planteada por Navarro \& Muñoz Garmendia (1998: 379), que consideraban factible la presencia de esta planta en Madrid. La población madrileña sería límite nororiental de la especie.

Consideramos que este endemismo ibérico debería incluirse en el catálogo madrileño de especies amenazadas, porque para los criterios UICN (2012) estaría clasificada en peligro crítico.

\section{Succisella carvalhoana (Mariz) Barksay}

ESPAÑA, Madrid: San Martín de Valdeiglesias, proximidades del Arroyo del Linar, hacia el límite con Cadalso de los Vidrios, 30TUK8264, 775 m., en prado muy húmedo, 15-IX-2016, J. Araújo Díaz de Terán (MAF 177430); ibidem, 28-IX-2018, G. López González (MAF 177431) (Fig. 6).

Endemismo ibérico restringido al centro y centrooeste peninsular (Devesa \& Amich, 2007: 308), incluido en la Lista Roja de la Flora Vascular Española con la categoría de Vulnerable (Bañares et al., 2008). En 2006 sólo se había confirmado su presencia en cuatro poblaciones del total de localidades españolas en las que había sido señalada, con un total de 1.100 individuos reproductivos (Amich \& Bernardos, 2006: 82-83).

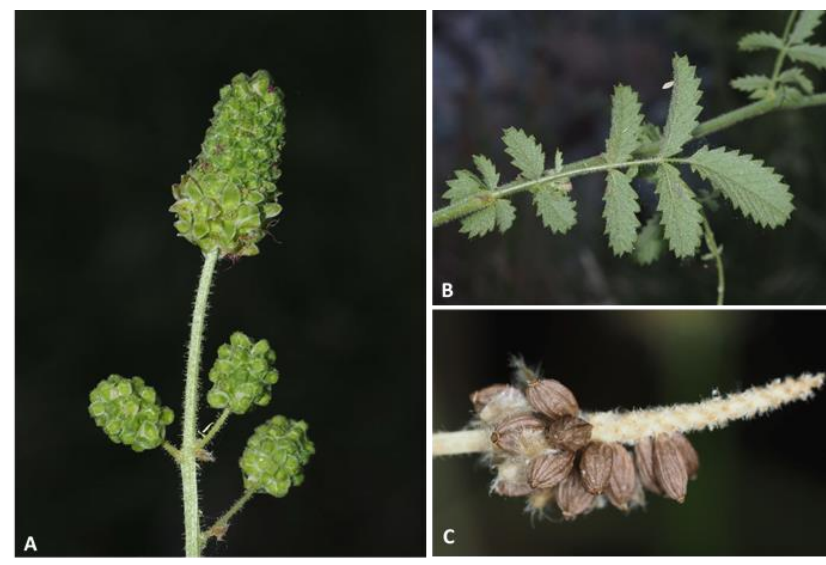

Figura 5. Sanguisorba hybrida en Cadalso de los Vidrios (Madrid). A- infrutescencia, B - detalle de hojas, C - frutos. Figure 5. Sanguisorba hybrida in Cadalso de los Vidrios (Madrid). A- infrutescence, $B$ - leaf detail, $C$ - fruits.

La población aquí descrita supera el centenar de individuos reproductivos, diseminados en unas 6 hectáreas de un prado muy húmedo con elementos de Arrhenatheretalia Tüxen 1931 (Rivas-Martínez et al., 2002) y formaciones compactas de Nardus stricta L. en los enclaves con mayor retención de agua. Se

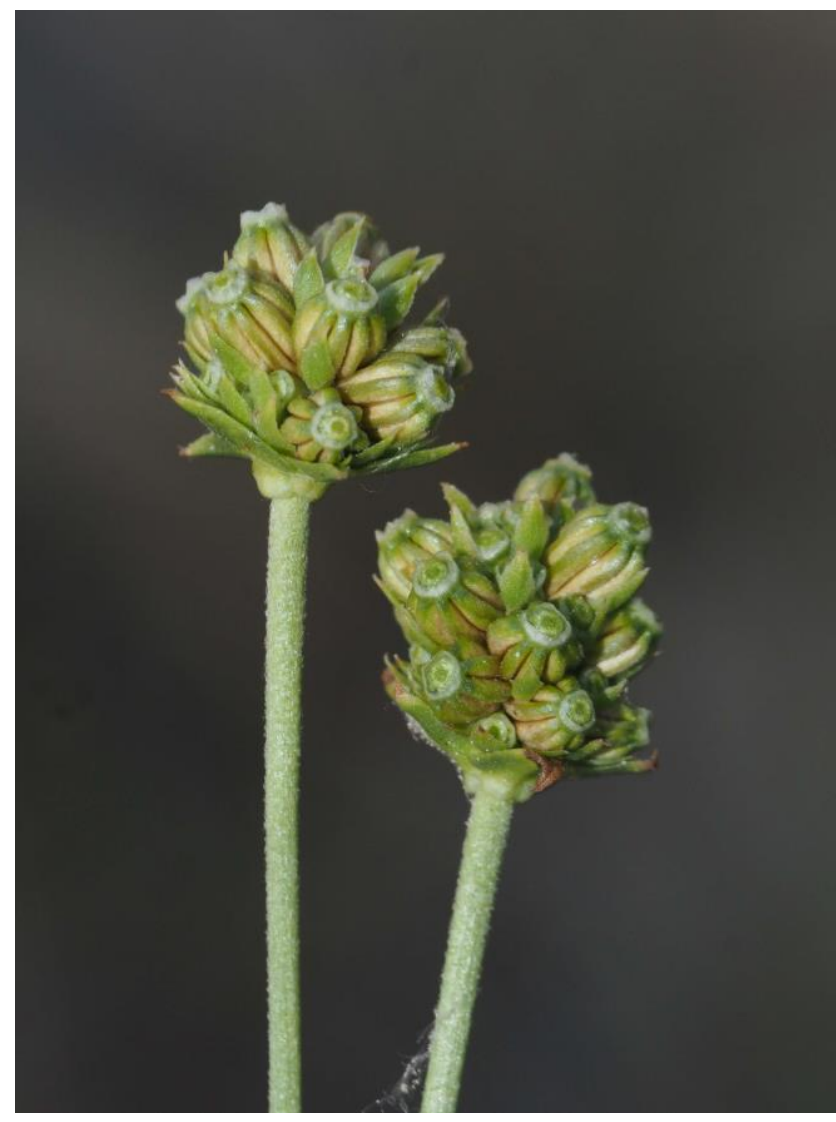

Figura 6. Succisella carvalhoana en San Martín de Valdeiglesias (Madrid), frutos.

Figure 6. Succisella carvalhoana in San Martín de Valdeiglesias (Madrid), fruits. 
había señalado previamente para Madrid (Devesa \& Amich, 2007: 308; López Jiménez, 2007: 66), la consulta de los datos de herbario (véase MA 628929) atribuyen las citas al municipio de Rozas de Puerto Real; esta población y la que se aporta ahora suponen las localidades más orientales conocidas para la especie. No se ha observado pastoreo en el lugar aquí señalado, hecho que junto al elevado número de individuos detectados puede suponer una ventaja de cara a su conservación frente a otras poblaciones que tienen su futuro comprometido por el sobrepastoreo, como ya han señalado en otras localidades Amich \& Bernardos (2006: 83). Estos prados son sometidos a siega mecanizada hacia finales de mayo o principios de junio, lo cual en apariencia no compromete la existencia de la especie en la zona por lo tardío de su fenología estivo-otoñal.

En aplicación de los criterios de la UICN (2012) se debería catalogar al menos en la categoría de Vulnerable.

\section{Agradecimientos}

Agradecemos a Pepe Pizarro y José Manuel Sobrados las facilidades para incluir el material en el herbario MAF, así como al personal del resto de herbarios consultados las facilidades proporcionadas para acceder a los datos. A Esther García Guillén por la ayuda para consultar el archivo del Real Jardín Botánico de Madrid. Por último a los amigos que nos han acompañado en las visitas a las localidades. $Y$ al editor y revisores anónimos que han mejorado considerablemente este texto.

Este trabajo se ha realizado de manera desinteresada por los autores sin financiación pública o privada.

\section{Bibliografía}

Abajo, A., Carmona, E., Escribano, R., Ortega, C., Rodríguez, A., Ruiz del Castillo, J. \& Ruiz de la Torre, J. (1982). Aproximación al catálogo de plantas vasculares de la provincia de Madrid. Madrid: Comunidad de Madrid, Consejería de Agricultura y Ganadería.

Amich, F. \& Bernardos, S. (2006). Succisella carvalhoana (Mariz) Baksay. In A. Bañares, G. Blanca, J. Güemes, J.C. Moreno \&. S. Ortiz (Eds.), Atlas y Libro Rojo de la Flora Vascular Amenazada de España. Adenda 2006 (pp. 82-83). Madrid: Dirección General para la Biodiversidad-Sociedad Española de Biología de la Conservación de Plantas.

Anthos (2019). Sistema de información de las plantas de España. Madrid: Real Jardín BotánicoCSIC, Fundación Biodiversidad. Recurso electrónico en http://www.anthos.es [Consulta realizada en $\mathrm{XI} / 2019]$.

Bañares, A., Blanca, G., Güemes, J., Moreno, J.C. \& Ortiz, S. (2008, Eds.). Lista roja 2008 de la Flora Vascular Española. Madrid: Dirección General de Medio Natural y Política Forestal (Ministerio de Medio Ambiente, y Medio Rural y Marino)-Sociedad Española de Biología de la Conservación de Plantas).
Baonza Díaz, J. (2007). Algunas plantas raras en la Comunidad de Madrid. Nuevas citas y revisión corológica. Botanica Complutensis, 31, 87-95.

Bartolomé, C., Álvarez, J., Vaquero, J., Costa Tenorio, M., Casermeiro, M.A., Giraldo, J. \& Zamora, J. (2005). Los tipos de hábitat de interés comunitario de España. Guía Básica. Madrid: Organismo Autónomo Parques Nacionales.

Benedí, C. (2019). Glebionis Cass. In C. Benedí, A. Buira, E. Rico, M.B. Crespo, A. Quintanar \& C. Aedo (Eds.), Flora Iberica 16(3) (pp. 1974-1979). Madrid: Real Jardín Botánico, C.S.I.C.

Biodiversidadvirtual (2019).

http://www.biodiversidadvirtual.org/herbarium/

[Consulta realizada 20/IX/2019].

Cardiel, J.M. (2013). Romulea Maratti. In E. Rico, M.B. Crespo, A. Quintanar, A. Herrero \& C. Aedo (Eds.), Flora lberica 20 (pp. 465-473). Madrid: Real Jardín Botánico, C.S.I.C.

Cebolla Lozano, C. \& Rivas Ponce, M.A. (1994). Atlas florae matritensis (Amaryllidaceae, Iridaceae, Liliaceae, Orchidaceae). Fontqueria, 41, 1-206.

Cirujano Bracamonte, S. \& Medina Domingo, L. (2002). Plantas acuáticas de las lagunas y humedales de Castilla-La Mancha. Madrid: Real Jardín BotánicoConsejo Superior de Investigaciones Científicas, Junta de Comunidades de Castilla-La Mancha.

Crespo, M.B. (2005). Epipactis Zinn. In C. Aedo \& A. Herrero (Eds.), Flora Iberica 21 (pp. 22-54). Madrid: Real Jardín Botánico, C.S.I.C.

Cutanda, V. (1861). Flora compendiada de Madrid y su provincia. Madrid: Imprenta Nacional.

Devesa, J.A. \& Amich, F.M. (2007). Succisella Beck. In J.A. Devesa, R. Gonzalo \& A. Herrero (Eds.), Flora Iberica 15 (pp. 305-310). Madrid: Real Jardín Botánico, C.S.I.C.

DOUE (1992). Directiva 92/43/CE del Consejo, de 21 de mayo de 1992, Relativa a la Conservación de los Hábitats Naturales y de la Fauna y Flora Silvestres. Núm. 206, de 22 de julio de 1992, pp. 750.

Fernández Carvajal, M.C. (1986). Clematis L. In S. Castroviejo, M. Laínz, G. López González, P. Montserrat, F. Muñoz Garmendia, J. Paiva \& L. Villar. (Eds.), Flora Iberica 1 (pp. 268-272). Madrid: Real Jardín Botánico, C.S.I.C.

Gbif (2019). Global biodiversity information facility. Acceso libre y gratuito a los datos de biodiversidad. www.gbif.net [Consulta realizada XI/2018].

Grijalbo, J. (2016 a). Flora de Madrid. Madrid: Ed. J. Grijalbo Cervantes.

Grijalbo, J. (2016 b). Flora de Madrid, Catálogo de plantas vasculares de la Comunidad de Madrid. http://javiergrijalbo.blogspot.com/p/flor.html [Consulta realizada XII/2018].

Inventario (1771). Inventario de todas las Plantas, Utensilios y Enseres del Real Jardín Botánico, hecho en el mes de Octubre de 1771 [Manuscrito]. Biblioteca histórica de Santa Cruz de Valladolid, Signatura: U/Bc Ms 109. http://uvadoc.uva.es/handle/10324/267 [Consulta realizada 9/XI/2018].

López Jiménez, N. (2007). Las plantas vasculares de la Comunidad de Madrid. Catálogo florístico. Claves dicotómicas y estudio detallado de la familia 
Compositae Giseke. Madrid: Tesis Doctoral. Facultad de Ciencias Biológicas, Universidad Complutense.

Martínez Labarga, J.M. (2014). Estudios corológicos de plantas vasculares en la cuenca media del Tajo. Madrid: Tesis doctoral, E.T.S. de Ingeniería de Montes, Forestal y del Medio Natural. Universidad Politécnica.

Martínez Labarga, J.M. (2016). Dos Astragalus L. (Leguminosae) a añadir a la flora de la Comunidad de Madrid. Acta Botánica Malacitana, 41, 258-260. http://dx.doi.org/10.24310/abm.v41i0.2467

Martínez Labarga, J.M. (2018). Confirmada la presencia de Garidella nigellastrum L. en Madrid. Acta Botánica Malacitana, 43, 141-142. http://dx.doi.org/10.24310/abm.v43i0.3512

Martínez Labarga, J.M., Araújo Díaz de Terán, J., Ramos Gutiérrez, I., Giraldo Barragán, C. \& Moreno Sáiz, J.C. (2017). Evolución de la población de la orquídea gigante (Himantoglossum robertianum) en la Comunidad de Madrid. Conservación Vegetal, 21, 7-11. http://dx.doi.org/10.15366/cv2017.21

Morales Valverde, R. (2003). Catálogo de plantas vasculares de la Comunidad de Madrid. Botanica Complutensis, 27, 31-70.

Navarro, C. \& Muñoz Garmendia, F. (1998). Sanguisorba L. In F. Muñoz Garmendia \& C. Navarro (eds.), Flora Iberica 6 (pp. 375-388). Madrid: Real Jardín Botánico, C.S.I.C.

Nieto Feliner, G. (2003). Eryngium L. In G. Nieto Feliner, S.L. Jury \& A. Herrero (Eds.), Flora Iberica 10 (pp. 36-60). Madrid: Real Jardín Botánico, C.S.I.C. Real Jardín Botánico - C.S.I.C.: Colecciones (2018). Herbario del Real Jardín Botánico, búsqueda por nombre científico. http://colecciones.rjb.csic.es/ [Consulta realizada XII/2018].

Rivas-Martínez, S., Díaz, T.E., Fernández González, F., Izco, J., Loidi, J., Lousã, M. \& Penas, A. (2002). Vascular plant communities of Spain and Portugal. Addenda to the syntaxonomical checklist of 2001. Part II. Itinera Geobotanica, 15 (1), 5-432; 15(2), 433-922.

Sivim (2018). Sistema de información de la vegetación Ibérica y Macaronésica. Universidad de Barcelona, Universidad del País Vasco, Universidad de Castilla-La Mancha (Toledo), Universidad de León. Ministerio de Educación y Ciencia. http://www.sivim.info/sivi/ [Consulta realizada $\mathrm{XI} / 2018]$.

The Plant List (2013). A working list of all plant species. Version 1.1. http://www.theplantlist.org/ [Consulta realizada $\mathrm{X} / 2018$ ].

Thiers, B. \& Tulig, M. (2019). Index Herbariorum: A global directory of public herbaria and associated staff. New York Botanical Garden's Virtual Herbarium. The New York Botanical Garden, New York. https://www.nybg.org/science-project/indexherbariorum-upgrade [Consulta realizada XI/2019].

UICN (2012). Categorías y Criterios de la Lista Roja de la UICN: Versión 3.1. Segunda edición. Gland, Suiza y Cambridge, Reino Unido: UICN. 